\title{
Spheroids scaling relations over cosmic time
}

\author{
Tommaso Treu \\ Department of Physics, University of California, Santa Barbara, CA 93106-9530, USA \\ email:tt@physics.ucsb.edu
}

\begin{abstract}
I report on recent measurements of two scaling relations of spheroids in the distant universe, the Fundamental Plane, and the relation between lensing velocity dispersion and stellar velocity dispersion. The joint analysis of the two scaling relations indicates that the most massive (above $\sim 10^{11.5} M_{\odot}$ ) spheroids are consistent with no evolution since $z \sim 1$ both in terms of star formation and internal structure. Furthermore their total mass density profile is on average well described by an isothermal sphere with no evidence for redshift evolution. At smaller masses the picture appears to be substantially different, as indicated by evidence for substantial recent star formation (as much as $20-40 \%$ of stellar mass formed since $z \sim 1$ ), and by hints of a reduced dark matter content at smaller masses. A larger sample of lenses extending to velocity dispersions below $200 \mathrm{kms}^{-1}$, and to redshifts above $>0.5$ is needed to verify these trends.
\end{abstract}

Keywords. gravitational lensing; stellar dynamics; galaxies: elliptical and lenticular, cD; galaxies: evolution galaxies: formation; galaxies: halos; (cosmology:) dark matter

\section{Introduction}

Spheroids (i.e. elliptical and lenticular galaxies, or collectively early-type galaxies) are observed to obey tight empirical scaling relations, i.e. correlations between observable properties. Well known examples of tight scaling relations are the Fundamental Plane (Dressler et al. 1987; Djorgovski \& Davis 1987; hereafter FP) and the $\mathrm{M}_{\mathrm{BH}}-\sigma$ relation (Ferrarese \& Merritt 2001; Gebhardt et al. 2001). The connection between observables involving dynamics, stellar populations, and the mass of the central black hole, indicates that mass assembly, star formation history, and nuclear activity are interconnected.

A common application of scaling relations in the distant universe is their use as "generalized standard rods". For example the FP is often used to derive the evolution of the effective mass to light ratio as a function of mass and redshift (Franx 1993; Treu et al. 1999). Assuming pure luminosity evolution this can then be converted into a star formation history (Treu et al. 2005a,b). Similarly the evolution of the $\mathrm{M}_{\mathrm{BH}}-\sigma$ relation can be used to quantify the relative growth of bulges and black holes over cosmic time (e.g. Shields et al. 2003; Woo et al. 2006). Here I will briefly report on recent progress in two areas: the evolution of the Fundamental Plane, and that of a less known scaling relation, i.e. the correlation between stellar velocity dispersion and total mass as measured by strong gravitational lensing.

\section{The Fundamental Plane}

By defining an effective mass $M$ in the terms of stellar velocity dispersion and effective radius ( $M=\mathrm{K}_{\mathrm{V}} \sigma^{2} R_{\mathrm{e}}$, where $\mathrm{K}_{\mathrm{V}}$ is the so-called virial coefficient) the $\mathrm{FP}$ can be seen as a scaling relation between $M$ and the effective mass-to-light ratio $M / L$. If one assumes that $M / L$ is proportional to the stellar mass-to-light ratio $M * / L$, the FP provides a very robust measurement of the evolution of $M * / L$ as a function of $M$. Note that this 

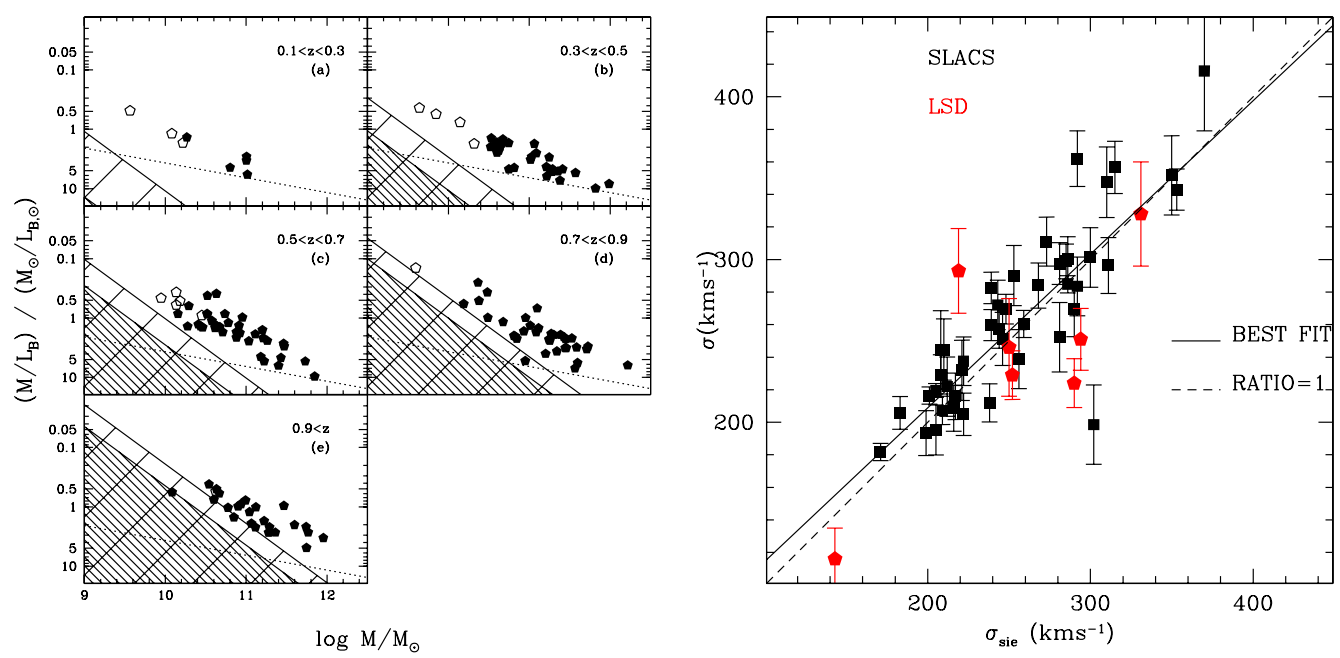

Figure 1. Left: Fundamental Plane as a function of redshift projected on the $M-M / L$ plane. The local relation is shown as a dotted line for comparison. Hatched regions are excluded by the magnitude limit. Although selection effects enhance the observed evolution at the low mass end, careful modeling shows that the evolutionary rate depends on mass, and that the intrinsic scatter of the FP increases with redshift. From Treu et al. 2005b. Right: Scaling relation between velocity dispersion of the stars $(\sigma)$, and that of the singular velocity dispersion ellipsoids that best fits the lensing constraints $\left(\sigma_{\mathrm{SIE}}\right)$, for the LSD and SLACS sample as of August 2006.

procedure does not assume a constant virial coefficient as a function of mass, but only that the virial coefficient be constant as a function of time at any given mass.

Observational studies are now capable of probing out to redshift $\sim 1$ with samples of over 100 objects, covering more than an order of magnitude in $M$. The main results are shown in Figure 1 (see also van der Wel et al. 2005 and di Serego et al. 2005). The FP evolves with redshift, in the sense that galaxies were brighter at high-z than today for a given mass. Evolution is slowest for the most massive objects, consistent with pure luminosity evolution if the most massive galaxies have the oldest stellar populations. In terms of assembly history, the fraction of stellar mass assembled below $z \sim 1$ is constrained to be of order $1 \%$ for the most massive objects $\left(M>10^{11.5} M_{\odot}\right)$, and up to $\sim 20-40 \%$ for galaxies of masses of order $10^{10} \mathrm{M}_{\odot}$. This is another manifestation of the concept of 'downsizing', sometimes called 'anti-hierarchical' behavior. Recent theoretical work suggests that AGN feedback could reconcile this trend with the hierarchical paradigm. Direct empirical tests of this mechanism are needed.

\section{A scaling relation measuring density profiles}

A recent explosion in the number of gravitational lenses with measured stellar velocity dispersion $\sigma$ (> 50; Bolton et al. 2005, 2006 and in prep.) allows one to study systematically the scaling of $\sigma$ with $\sigma_{\mathrm{SIE}}$, i.e. the velocity dispersion of the singular isothermal ellipsoid that best fits the lensing geometry. The latter is a very robust, and almost model independent, measure of mass within the critical line, i.e. approximately within a cylinder of radius the Einstein Radius. This is typically in the range $0.5-5 R_{\mathrm{e}}$. Since $\sigma$ measures the mass inside $\sim R_{\mathrm{e}} / 8$, this scaling relation effectively provides a measurement of the slope of the mass density profile. Modulo small corrections due to anisotropy, projection 
effects, and the precise location of the Einstein Radius with respect to the effective radius, a ratio of unity indicates an isothermal total mass density profile (see Treu \& Koopmans 2002, 2004, Koopmans \& Treu 2003, Koopmans et al. 2006, for detailed modeling). This is observed to be generally the case within the errors, with few outliers (Figure 1).

This tight scaling relation can be seen as the result of a "bulge-halo conspiracy", because neither of the two components has an isothermal profile and yet the sum of the two is very close to it. Remarkably, no significant evolution of the total mass density slope is detected. Whatever mechanism produces the conspiracy it seems to be at work at high-z for these massive galaxies. Note that SLACS lens galaxies are indistinguishable from a control sample of identically selected non-lens early-type galaxies within the current observational uncertainties (Treu et al. 2006), and therefore these results can be generalized to the entire population of massive early-type galaxies. From an observational point of view, there is room for improvement, especially at $z>0.5$, where the samples are still pitifully small and SDSS runs out of lenses. A comparable sample of $\sim 50$ lenses at $z>0.5$ would help enormously to understand evolutionary trends.

\subsection{Does the dark matter content depend on mass?}

Finally, I will briefly discuss the fraction of dark matter as a function of mass. There is convincing evidence that the most massive early-type galaxies have dark matter halos more extended than the stellar component, but the evidence is not quite as compelling for less massive galaxies. Determining the relative contribution of dark and stellar matter as a function of mass is important for a number of reasons, e.g. understanding the efficiency of star formation as a function of mass, and the interplay between baryons and dark matter. In terms of scaling relations, one of the possible explanations of the so-called "tilt" of the Fundamental Plane is a systematic increase with effective mass of the dark matter fraction inside some fiducial radius (e.g. Ciotti et al. 1996).

A joint lensing and dynamical analysis of the SLACS+LSD sample (Treu \& Koopmans 2004; Koopmans et al. 2006) provides a robust measurement of the fraction of dark matter at the Einstein Radius for each lens. Since lenses span a range in velocity dispersions (a proxy for mass) and in Einstein Radii (expressed in units of the effective radius) this appears to be a promising way to attack this problem. The promise of this method is illustrated in Figure 2. In the left panel, I show the fraction of dark matter inside the Einstein Radius as derived from the lensing and dynamical analysis as a function of the Einstein Radius in units of the effective radius. If the dark matter halo is more spatially extended than the light, the fraction of dark matter will be increasing with radius. Therefore, this effect has to be taken into account, in order to study trends with velocity dispersion. One way to decouple the dependency on radius from that on mass is to look at deviations from a simple reference model, known to work quite well for the most massive systems (e.g. Treu \& Koopmans 2002). This simple model consists of an isothermal total mass profile, including a Jaffe (1983) profile representing the stars (the remaining mass is assumed to be dark matter). The Jaffe profile is normalized so that the fraction of dark matter is zero at the center. Thus, the fraction of dark matter is a universal function of radius in units of the effective radius, shown by a solid line in the figure. In the right panel, I show the dark matter deficit/excess with respect to the simple reference model. Tantalizingly, it seems that the simple model works very well for the most massive systems, while the least massive systems appear to show a deficit of dark matter with respect to the model. Drawing quantitative conclusions from this preliminary analysis is premature, as a comprehensive analysis of a much enlarged sample of lenses is underway. 

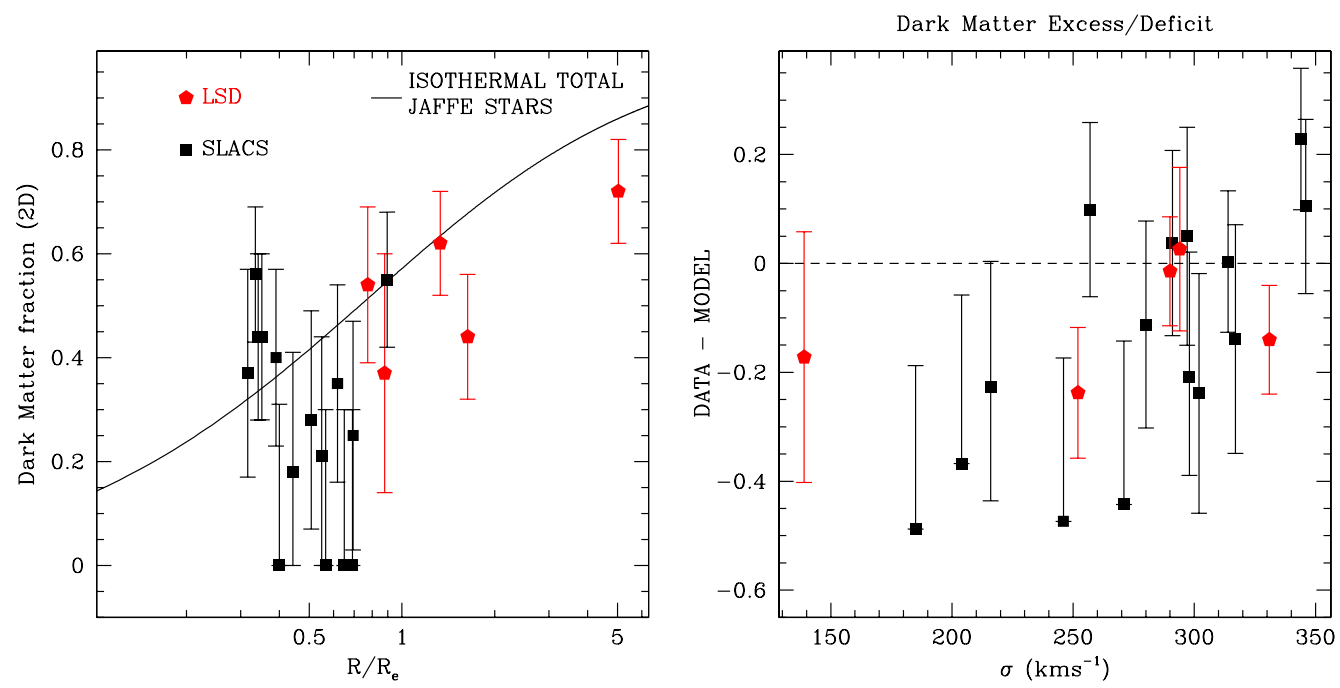

Figure 2. Left: Dark matter fraction inside the cylinder of radius equal to the Einstein Radius compared to the expectation of a model where the total mass distribution is isothermal and the stars follow a Jaffe profile. Right: dark matter excess/deficit with respect to the model.

\section{Acknowledgements}

I would like to acknowledge the many contributions to this work from my friends and collaborators Richard Ellis, Léon Koopmans, Adam Bolton, Scott Burles, and Leonidas Moustakas. This research is supported by NASA through STScI grants GO-10174, GO10494, AR-9920, AR-09960. Many thanks to the AAS and the IAU for travel grants and to the organizers of IAU235 for inviting me.

\section{References}

Bolton, A., Burles, S. M., Koopmans, L. V. E., Treu, T., \& Moustakas, L. M. 2005, ApJ, 624, L21

Bolton, A., Burles, S. M., Koopmans, L. V. E., Treu, T., \& Moustakas, L. M. 2006, ApJ, 638, 703

Ciotti, L., Lanzoni, B., \& Renzini A. 1996, MNRAS, 282, 1

di Serego Alighieri, S. et al. 2005, A\&A, 442, 125

Djorgovksi S. G., Davis M., 1987, ApJ, 313, 59

Dressler, A., Lynden-Bell, D., Burstein, D., Davies, R. L., Faber, S. M., Terlevich, R, \& Wegner G. $1987, A p J, 313,42$

Franx, M. 1993, PASP, 105, 1058

Jaffe, W. 1983, MNRAS, 202, 995

Koopmans, L. V. E., \& Treu, T. 2002, ApJ, 568, L5

Koopmans, L. V. E., \& Treu, T. 2003, ApJ, 583, 606

Koopmans, L. V. E., Treu, T., Bolton, A. S., Burles, S., \& Moustaks, L. A. 2006, ApJ, 649, 599

Shields, G. A., Gebhardt, K., Salviander, S., Wills, B. J., Xie, B., Brotherton, M. S., Yuan, J., \& Dietrich, M. 2003, ApJ, 583, 124

Treu, T., \& Koopmans, L. V. E. 2002, ApJ, 575, 87

Treu, T., \& Koopmans, L. V. E. 2003, MNRAS, 343, L29

Treu, T., \& Koopmans, L. V. E. 2004, ApJ, 611, 739

Treu, T., Ellis, R. S., Liao, T.X., \& van Dokkum P.G. 2005, ApJ, 622, L5

Treu, T., et al. 2005, ApJ, 633, 174 
Treu, T., Koopmans, L. V. E., Bolton, A. S., Burles, S. C., \& Moustakas, L. A. 2006, ApJ, 640, 662

Treu, T., Stiavelli, M., Casertano, S., Møller, P., \& Bertin, G. 1999, MNRAS, 308, 1037

van der Wel, A., Franx, M., van Dokkum, P. G., Rix, H.-W., Illingworth, G. D., \& Rosati, P. 2005, ApJ, 631, 145

Woo, J.-H., Treu, T., Malkan, M. A., \& Blandford, R. D. 2006, ApJ, 645, 900

\section{Discussion}

Mike Hudson: Have you compared the stellar masses (inferred from the SF histories consistent with the evolution of the fundamental plane) to the total masses (inferred from dynamics and lensing) to see if they are consistent?

Tomasso Treu: Yes, within the uncertainties, the stellar M/L from the fundamental plane are consistent with that from the lensing and dynamical models. The total M/L is of course higher.

Marc VerheiJen: Does the bulge/halo conspiracy indicate an evolution of the mass distribution in galaxies? At $\mathrm{z}=0$, the bulge in Sa galaxies "breaks" the bulge/halo conspiracy suggesting that mass follows light. Is this the case at high redshift?

Tomasso Treu: It's difficult to draw a direct comparison. 1) E/SO are not Sa galaxies, so the comparison should be made with other samples 2) at high $\mathrm{z}$, we do not have that much spatial resolution, so the total slope of the mass profile that we measure is intended as an average effective slope on scales of order $1-10 \mathrm{kpc}$ or so. Things will improve in the next couple of years, as we are getting $2 \mathrm{D}$ spectroscopy on the nearest lenses.

Mordecai Mac Low: Downsizing can be explained by faster star formation in larger galaxies. Large galaxies are more gravitationnally unstable, so this may be plausible (Li, Mac Low and Klessen 2006).

Tomasso Treu: I agree, but you also need to prevent further gas cooling and star formation at a later time in the larger galaxies. 